\title{
The design of an optimal running curve for train operation based on a novel parameterization method aiming to minimize the total energy consumption
}

\author{
V. D. Doan, S. Watanabe \& T. Koseki \\ University of Tokyo, Japan
}

\begin{abstract}
This paper describes a new approach for creating an optimal running curve of a train with the aim of minimizing total energy consumption, given the constraints of running time, limited motive force and limited train acceleration. A novel parameterization method of the running curve is proposed for mathematically demonstrating the relationship between the speed of the train and the position of the train. Then, the total energy consumption and the above constraints are formulated as functions depending on the parameters of the running curve. From this scenario, a constrained optimization problem is considered for minimizing the total energy consumption. After that, an effective method to obtain the solution of this optimization problem is presented. The effectiveness of the proposed approach is verified by computer simulations.

Keywords: train operation, energy-saving, total energy consumption, optimal running curve, parameterization method, optimization.
\end{abstract}

\section{Introduction}

Railway transportation is facing increasing pressure to reduce its energy consumption due to increasing concerns for environment issues. Therefore, strategies in effectively using energy are becoming more important than before, and studies on train operation to reduce the total energy consumption are crucial. Since the running curve directly affects the total energy consumption, many researchers have considered how to obtain an optimal running curve so that this energy consumption is minimized. Those researches can be grouped into two 
categories as coasting control and general control (e.g. Lu [1]). In coasting control, control algorithms are proposed to find appropriate coasting points at which the traction motors are completely switched off. For example, Genetic algorithm is implemented to search for these points (e.g. Chang and Sim [2]); and Artificial Neural Network is applied to search for optimal coasting speed to minimize the social cost (e.g. Chuang et al. [3]). Meanwhile, general control seeks for the optimal running curve by searching the train speed at various positions of all three modes of train operation (i.e. Powered, Coasting, and Braking modes). There are numerous algorithms used to obtain these optimal control sequences of train such as Pontryagin's maximum principle (e.g. Liu and Golovitcher [4]), Genetic Algorithm (e.g. Liu and Li [5]), and Dynamic Programming (e.g. Ko et al. [6]). For those approaches, the optimal running curve is obtained by searching for the speed of train at various positions; furthermore, at each step of searching process, we have to solve nonlinear differential equation of train that is quite complicated, so those algorithms are in computational burden due to their generic nature. In this research, we suggest a new approach for searching an optimal running curve. Instead of finding speed of train at various discrete positions of train, we find mathematical functions to demonstrate speed-position relationship. With this approach, we do not need to solve that nonlinear differential equation. Besides, for passenger's comfort, limited train acceleration is also considered. The effectiveness of the parameterization method is verified by computer simulation.

The remains of this paper are outlined as follows: Section 2 derives formula of the total energy consumption of train. Section 3 proposes a novel parameterization method of the running curve and a method to choose parameterization functions. Then, Section 4 establishes the general constrained optimization problem based on the parameterization method. Section 5 develops an effective method to solve the optimization problem stated above. Section 6 presents simulation results. Section 7 presents our conclusions.

\section{Mathematical model of train operation}

\subsection{Dynamic equations of the train}

The dynamic equation of the train follows Newton's third law [6]:

$$
\left\{\begin{array}{c}
\frac{d v}{d t}=\frac{1}{M}\left[F_{m}-F_{m e c h}(v)-R(x, v)\right] \\
\frac{d x}{d t}=v
\end{array}\right.
$$

where, $x$ - position of the train; $v$ - speed of the train; $\frac{d v}{d t}$ - acceleration of the train; $M$ - total mass of the train; $F_{m}$ - motive force of the train; $F_{m e c h}(v)-$ mechanical braking force; $R(x, v)$ - total resistances of train $(R(x, v)$ is a function of $x$ and $v$ ). 


\subsection{Energy equations of the train}

Powered Mode: $F_{m}>0 ; F_{\text {mech }}=0$

Powered energy: $E_{a c c}=\frac{1}{\eta} \int_{0}^{T_{1}} F_{m} v d t$;

Inverter energy loss: $E_{\text {acc_inv }}=\left(\frac{1}{\eta}-1\right) \int_{0}^{T_{1}} F_{m} v d t$;

Resistance loss: $E_{\text {acc_res }}=\int_{0}^{T_{1}} R(x, v) v d t$.

Where, $\eta$ is inverter efficiency; $T_{1}$ is time for Powered mode.

Coasting Mode: $F_{m}=0 ; F_{\text {mech }}=0$

No input energy is applied to the train.

Resistance loss: $E_{\text {coa_res }}=\int_{T_{1}}^{T_{2}} R(x, v) v d t$

$T_{2}-T_{1}$ is time for Coasting mode.

Braking Mode: $F_{m}<0 ; F_{\text {mech }} \geq 0$

Regenerative energy: $E_{\text {reg }}=-\eta \int_{T_{2}}^{T} F_{m} v d t$

Mechanical braking energy: $E_{\text {mech }}=\int_{T_{2}}^{T} F_{\text {mech }}(v) v d t$

Energy inverter loss: $E_{\text {dec_inv }}=-(1-\eta) \int_{T_{2}}^{T} F_{m} v d t$

Resistance loss: $E_{\text {dec_res }}=\int_{T_{2}}^{T} R(x, v) v d t$; where, $T$ is the running time.

We can mathematically prove that:

$$
\begin{gathered}
E_{\text {total }}=E_{\text {acc }}-E_{\text {reg }}=\frac{1}{\eta} \int_{0}^{T_{1}} F_{m} v d t+\eta \int_{T^{2}}^{T} F_{m} v d t \\
=\left(\frac{1}{\eta}-\eta\right) \int_{0}^{T_{1}} F_{m} v d t+\eta\left(\int_{0}^{T_{1}} F_{m} v d t+\int_{T_{2}}^{T} F_{m} v d t\right) \\
=\left(\frac{1}{\eta}-\eta\right) \int_{0}^{T_{1}} F_{m} v d t+\eta\left(\int_{T_{2}}^{T} F_{\text {mech }}(v) v d t+\int_{0}^{d} R(x, v) d x\right)
\end{gathered}
$$

where, $d_{1}, d_{2}$, and $d$ are respectively notch-off speed position, coasting-off position, and distance between two stations.

\section{A novel parameterization method of running curve}

\subsection{Parameterization of running curve}

As stated above, the operation of train is described by a Running Curve including three modes: (1) Powered mode, (2) Coasting mode, and (3) Braking mode. If we assume that each of the modes can be modelled by a function relationship, we have three functions to demonstrate the three modes: 
Table 1: Parameterization of running curve.

\begin{tabular}{|l|l|c|}
\hline \multicolumn{1}{|c|}{ Mode } & \multicolumn{1}{c|}{ Function } & No. \\
\hline Powered & $v=y_{1}\left(\boldsymbol{p}_{1}, x\right),\left(0 \leq x \leq d_{1}\right)$ & $(3.1)$ \\
\hline Coasting & $v=y_{2}\left(\boldsymbol{p}_{2}, x\right),\left(d_{1} \leq x \leq d_{2}\right)$ & $(3.2)$ \\
\hline Braking & $v=y_{3}\left(\boldsymbol{p}_{3}, x\right),\left(d_{2} \leq x \leq d\right)$ & $(3.3)$ \\
\hline
\end{tabular}

where, functions $y_{1}, y_{2}$, and $y_{3}: R \rightarrow R$ are assumed to be continuous and differentiable in the ranges of $\left[0 ; d_{1}\right],\left[d_{1} ; d_{2}\right]$, and $\left[d_{2} ; d\right]$, respectively. The row vectors $\boldsymbol{p}_{1}, \boldsymbol{p}_{2}, \boldsymbol{p}_{3}$ are respectively parameters of the functions $y_{1}, y_{2}, y_{3}$. In other words, the running curve can be parameterized by $d_{1}, d_{2}$ and $\boldsymbol{p}_{1}, \boldsymbol{p}_{2}, \boldsymbol{p}_{3}$.

\subsection{Choosing parameterization functions}

We always have: $a=\frac{d v}{d t}=v \frac{\partial v}{\partial x} \rightarrow v d v=a d x \rightarrow \frac{v^{2}}{2}=\int a d x$. If we assume that Acceleration-Position of Train can be described: $a=a(x)$, and let $A(x)=\int a d x$ be the primitive function of $a(x)$, Speed-Position relationship of Train can be described: $v(x)=\sqrt{2 A(x)}$. In practical train operation, there are several general shapes of $a(x)$; for example, some general shapes of $a(x)$ can be:

$$
\begin{gathered}
a(x)=C_{0}+C_{1} \sqrt{x}+C_{2} \sqrt[3]{x}+\cdots \\
a(x)=C_{0}+\frac{C_{1}}{x}+\frac{C_{2}}{x^{2}}+\cdots \\
a(x)=C_{0}+C_{1} x+C_{2} x^{2}+\cdots
\end{gathered}
$$

where, $C_{0}, C_{1}, C_{2}$ are coefficients of these functions. Thus, we can choose appropriate functions for demonstrating the running curve based on functions derived as below (here, $C=$ const):

$$
\begin{aligned}
& v(x)=\sqrt{2\left(C+C_{0} x+\frac{2 C_{1}}{3} x^{\frac{3}{2}}+\frac{3 C_{1}}{4} x^{\frac{4}{3}}+\cdots\right)} \\
& v(x)=\sqrt{2\left(C+C_{0} x+C_{1} \log (x)-\frac{C_{2}}{x}+\cdots\right)} \\
& v(x)=\sqrt{2\left(C+C_{0} x+\frac{C_{1}}{2} x^{2}+\frac{C_{2}}{3} x^{3}+\cdots\right)}
\end{aligned}
$$




\section{Optimization problem based on parameterization method for finding optimal running curve}

\subsection{Energy consumption: cost function}

From eqn (1),

$$
F_{m} d t=F_{\text {mech }}(v) d t+R(x, v) d t+M d v
$$

Since $F_{m e c h}=0$ in the range $\left[0 ; T_{1}\right]$, multiply both sides by $v$, then get integrals:

$$
\int_{0}^{T_{1}} F_{m} v d t=\int_{0}^{T_{1}} R(x, v) v d t+\int_{v(0)}^{v\left(T_{1}\right)} M v d v=\int_{0}^{d_{1}} R(x, v) d x+M \frac{v_{1}^{2}}{2}
$$

Substitute eqn (3.1) into eqn (4.2), we get:

$$
\int_{0}^{T_{1}} F_{m} v d t=\int_{0}^{d_{1}} R\left(x, y_{1}\left(\boldsymbol{p}_{1}, x\right)\right) d x+M \frac{\left[y_{1}\left(\boldsymbol{p}_{1}, d_{1}\right)\right]^{2}}{2}
$$

On the other hand,

$$
\int_{0}^{d} R(x, v) d x=\int_{0}^{d_{1}} R(x, v) d x+\int_{d_{1}}^{d_{2}} R(x, v) d x+\int_{d_{2}}^{d} R(x, v) d x
$$

Substitute eqn (3.1), eqn (3.2), and eqn (3.3) into eqn (4.4), then we get:

$$
\begin{gathered}
\int_{T_{2}}^{T} F_{\text {mech }}(v) v d t+\int_{0}^{d} R(x, v) d x=\int_{d_{2}}^{d} F_{\text {mech }}\left(y_{3}\left(\boldsymbol{p}_{3}, x\right)\right) d x+ \\
\int_{0}^{d_{1}} R\left(x, y_{1}\left(\boldsymbol{p}_{1}, x\right)\right) d x+\int_{d_{1}}^{d_{2}} R\left(x, y_{2}\left(\boldsymbol{p}_{2}, x\right)\right) d x+\int_{d_{2}}^{d} R\left(x, y_{3}\left(\boldsymbol{p}_{3}, x\right)\right) d x
\end{gathered}
$$

From eqn (2), eqn (4.3), and eqn (4.5), we can state that the total energy consumption $E_{\text {total }}$ is a function of $d_{1}, d_{2}$, and $\boldsymbol{p}_{1}, \boldsymbol{p}_{2}, \boldsymbol{p}_{3}$ as follows:

$$
E_{\text {total }}=f\left(d_{1}, d_{2}, \boldsymbol{p}_{1}, \boldsymbol{p}_{2}, \boldsymbol{p}_{3}\right)
$$

\subsection{Equality constraints}

\subsubsection{Constraint of running time}

We always get: $v=\frac{d x}{d t} \rightarrow d t=\frac{d x}{v}$. Integral both sides in the time range $[0 ; T]$ : 


$$
\begin{gathered}
T=\int_{0}^{T} d t=\int_{0}^{x\left(T_{1}\right)} \frac{d x}{v}+\int_{x\left(T_{1}\right)}^{x\left(T_{2}\right)} \frac{d x}{v}+\int_{x\left(T_{2}\right)}^{x(T)} \frac{d x}{v}=\int_{0}^{d_{1}} \frac{d x}{v}+\int_{d_{1}}^{d_{2}} \frac{d x}{v}+\int_{d_{2}}^{d} \frac{d x}{v} \\
=\int_{0}^{d_{1}} \frac{d x}{y_{1}\left(\boldsymbol{p}_{1}, x\right)}+\int_{d_{1}}^{d_{2}} \frac{d x}{y_{2}\left(\boldsymbol{p}_{2}, x\right)}+\int_{d_{2}}^{d} \frac{d x}{y_{3}\left(\boldsymbol{p}_{3}, x\right)}
\end{gathered}
$$

\subsubsection{Boundary constraints of parameterization functions}

Because the speed of the train is continuous through each of the modes, we get:

$$
\begin{gathered}
v_{1}=\left(y_{1}\left(\boldsymbol{p}_{1}, x\right)\right)_{x=d_{1}}=\left(y_{2}\left(\boldsymbol{p}_{2}, x\right)\right)_{x=d_{1}} \\
v_{2}=\left(y_{2}\left(\boldsymbol{p}_{2}, x\right)\right)_{x=d_{2}}=\left(y_{3}\left(\boldsymbol{p}_{3}, x\right)\right)_{x=d_{2}} \\
v(0)=\left(y_{1}\left(\boldsymbol{p}_{1}, x\right)\right)_{x=0}=0 ; v(d)=\left(y_{3}\left(\boldsymbol{p}_{3}, x\right)\right)_{x=d}=0
\end{gathered}
$$

From eqn (4.7), eqn (4.8), eqn (4.9), and eqn (4.10), we arrange all of equalities:

$$
\boldsymbol{H}\left(d_{1}, d_{2}, \boldsymbol{p}_{1}, \boldsymbol{p}_{2}, \boldsymbol{p}_{3}\right)=\mathbf{0}
$$

\subsection{Inequality constraints}

\subsubsection{Constraint of Input Motive Force}

From eqn (1), we can obtain:

$$
F_{m}=F_{m e c h}(v)+M v \frac{\partial v}{\partial x}+R(x, v)
$$

Since Input Motive Force is limited by $F_{\text {limit }}(v)$, we get:

$$
\left|F_{m}\right|=\left|F_{m e c h}(v)+M v \frac{\partial v}{\partial x}+R(x, v)\right| \leq\left|F_{\text {limit }}(v)\right|
$$

\subsubsection{Constraint of acceleration and speed of train}

We always have: $a=\frac{d v}{d t}=v \frac{\partial v}{\partial x}$. Since acceleration and speed of train are respectively limited by $a_{\text {limit }}$ and $v_{\text {limit }}$, we get:

$$
v \leq v_{\text {limit }} ;|a|=\left|v \frac{\partial v}{\partial x}\right| \leq a_{\text {limit }}
$$


If we sample several points of eqn (4.13) and eqn (4.14) in the range of $[0 ; d]$, we obtain inequalities that can be arranged as follows:

$$
\boldsymbol{G}\left(d_{1}, d_{2}, \boldsymbol{p}_{1}, \boldsymbol{p}_{2}, \boldsymbol{p}_{3}\right) \leq \mathbf{0}
$$

From eqn (4.6), eqn (4.11), and eqn (4.15), we obtain the optimization problem:

Cost function:

$$
\begin{array}{ll}
\text { Cost function: } & E_{\text {total }}=f(\boldsymbol{x}) \\
\text { Equalities: } & \boldsymbol{H}(\boldsymbol{x})=\mathbf{0} \\
\text { Inequalities: } & \boldsymbol{G}(\boldsymbol{x}) \leq \mathbf{0} \\
\text { Variables: } & \boldsymbol{x}=\left(d_{1}, d_{2}, \boldsymbol{p}_{1}, \boldsymbol{p}_{2}, \boldsymbol{p}_{3}\right)^{T}
\end{array}
$$$$
\text { Inequalities: } \quad \boldsymbol{G}(\boldsymbol{x}) \leq \mathbf{0}
$$

\section{An effective optimization algorithm for general optimization problems}

For solving the constrained optimization problem eqns (4.16), this optimization problem is transformed to the unconstrained optimization problem. Augmented Lagrange algorithm should be used (e.g. Berhe [7]), so the latter objective function is:

$$
\begin{gathered}
F(\boldsymbol{x}, \boldsymbol{u}, \boldsymbol{v})=f(\boldsymbol{x})+\mu \sum_{i=1}^{m}\left(\max \left\{g_{i}(\boldsymbol{x})+\frac{u_{i}}{2 \mu}, 0\right\}\right)^{2}-\sum_{i=1}^{m} \frac{u_{i}^{2}}{4 \mu}+ \\
\sum_{j=1}^{p} v_{j} h_{j}(\boldsymbol{x})+\mu \sum_{j=1}^{p} h_{j}^{2}(\boldsymbol{x})
\end{gathered}
$$

where, $\mu$ is the penalty coefficient $(\mu>0) ; u$ and $v$ are respectively Lagrange multipliers of $G(x)$ and $H(x)$. Vectors: $x, u, v, G$ and $H$ are expanded as below:

$$
\boldsymbol{x}=\left[\begin{array}{c}
x_{1} \\
x_{2} \\
\vdots \\
x_{n}
\end{array}\right] ; \boldsymbol{u}=\left[\begin{array}{c}
u_{1} \\
u_{2} \\
\vdots \\
u_{m}
\end{array}\right] ; \boldsymbol{v}=\left[\begin{array}{c}
v_{1} \\
v_{2} \\
\vdots \\
v_{p}
\end{array}\right] ; \boldsymbol{G}(\boldsymbol{x})=\left[\begin{array}{c}
g_{1}(\boldsymbol{x}) \\
g_{2}(\boldsymbol{x}) \\
\vdots \\
g_{m}(\boldsymbol{x})
\end{array}\right] ; \boldsymbol{H}(\boldsymbol{x})=\left[\begin{array}{c}
h_{1}(\boldsymbol{x}) \\
h_{2}(\boldsymbol{x}) \\
\vdots \\
h_{p}(\boldsymbol{x})
\end{array}\right]
$$

A function $\operatorname{VIOL}(x)$ is defined as a measure of constraint violations as bellow:

$$
\operatorname{VIOL}(\boldsymbol{x})=\max \left\{g_{i}(\boldsymbol{x}),\left|h_{j}(\boldsymbol{x})\right|: i=1,2, \ldots, m ; j=1,2, \ldots, p\right\}
$$

Augmented Lagrange algorithm is described in Figure 1. 


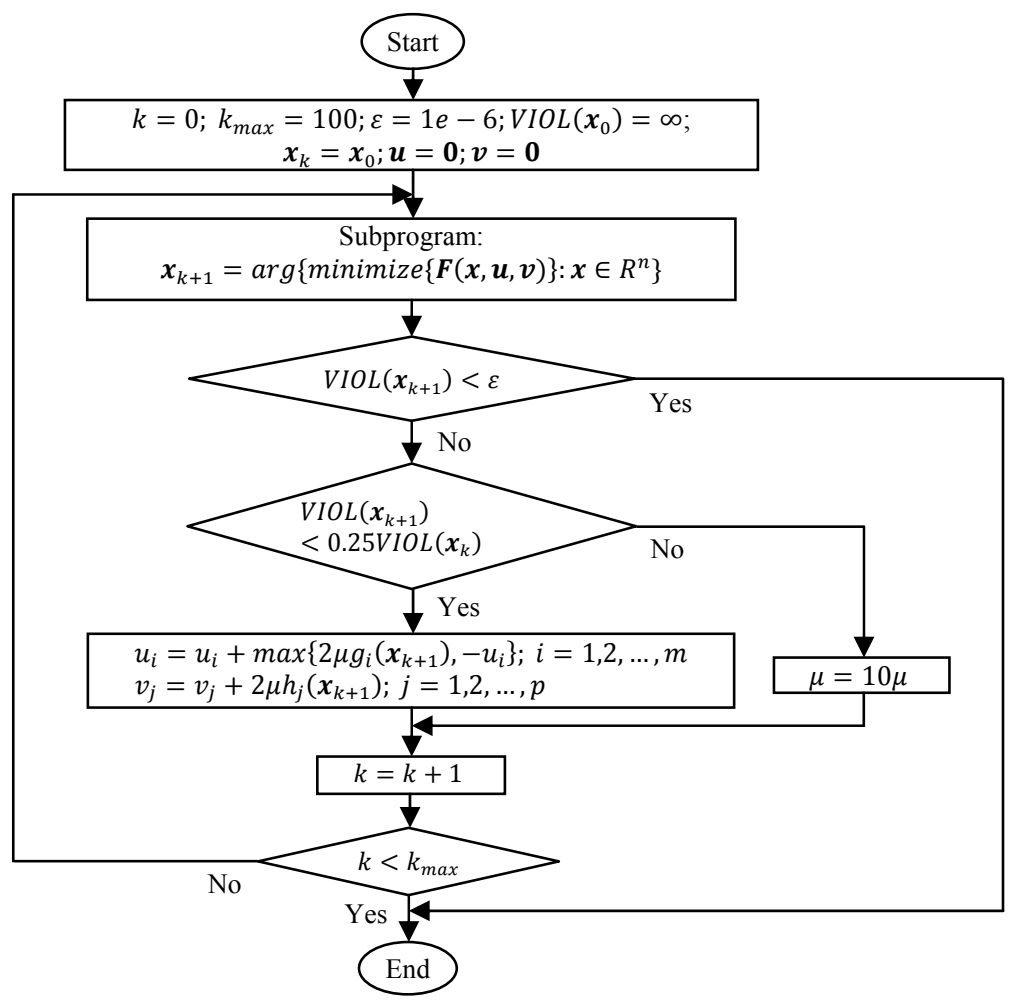

Figure 1: Augmented Lagrange algorithm.

As stated in Figure 1, the subprogram: minimize $\{\mathcal{F}(\boldsymbol{x})=F(\boldsymbol{x}, \boldsymbol{u}, \boldsymbol{v}): \boldsymbol{x} \in$ $\left.R^{n}\right\}$ needs calling at each iterative step of $k$. In general, an iterative-optimization process to find the optimal point of this problem follows the below equation:

$$
\boldsymbol{x}_{l+1}=\boldsymbol{x}_{l}+\alpha_{l+1} \boldsymbol{s}_{l+1} ; l=0,1,2, \ldots
$$

In each iterative step, the moving direction $\left(\boldsymbol{s}_{l+1} \in R^{n}\right)$ and the moving step $\left(\alpha_{l+1} \in R\right)$ are appropriately determined in order to guarantee convergence of the algorithm and its convergence speed. For calculating these values, the method of "Cleft-overstep" by perpendicular direction should be used (e.g. Nguyen and Bui [8]).

\section{A) The moving direction $\boldsymbol{s}_{l+1}$}

The moving direction $\boldsymbol{s}_{l+1}$ is calculated by a linear combination of latest inversegradient direction vectors of as below:

$$
\boldsymbol{s}_{l+1}=\sum_{i=1}^{r} \theta_{i} \nabla_{l-i+1}, r \leq n
$$


where, $\theta_{i} \in R$ is unknown coefficient satisfying the condition: $\sum_{i=1}^{r} \theta_{i}=1$; $\nabla_{l-i+1}=-\operatorname{grad} \mathcal{F}\left(x_{l-i+1}\right) ; \operatorname{grad}(\mathcal{F}(\boldsymbol{x}))$ - the gradient vector of the optimization function $\mathcal{F}(\boldsymbol{x}) ; r$ is the number of the latest gradient vectors stored in optimization process.

Since the moving direction $s_{l+1}$ is chosen so that the vector $s_{l+1}$ is orthogonal with $(r-1)$ vectors of $\boldsymbol{\nabla}_{l}-\boldsymbol{\nabla}_{l-1}, \boldsymbol{\nabla}_{l-1}-\boldsymbol{\nabla}_{l-2}, \ldots, \boldsymbol{\nabla}_{l-r+2}-\boldsymbol{\nabla}_{l-r+1}$, the following conditions is satisfied:

$$
\left\langle\boldsymbol{s}_{l+1}, \boldsymbol{\nabla}_{l-j+1}-\boldsymbol{\nabla}_{l-j}\right\rangle=0 ; j=1,2, \ldots, r-1
$$

where, $\langle\boldsymbol{a}, \boldsymbol{b}\rangle$ is denoted as the scalar product of two vectors $\boldsymbol{a}$ and $\boldsymbol{b}$.

Thus, the unknown parameters $\theta_{i}(i=1,2, \ldots, r)$ can be obtained by solving the following linear algebra equation:

$$
\left\{\begin{array}{c}
\sum_{i=1}^{r} \theta_{i}=1 \\
\sum_{i=1}^{r} \theta_{i}\left\langle\nabla_{l-i+1}, \nabla_{l-j+1}-\nabla_{l-j}\right\rangle=0 ; j=1,2, \ldots, r-1
\end{array}\right.
$$

Hence, the moving direction $\boldsymbol{s}_{l+1}$ is completely determined.

B) The Moving Step $\alpha_{l+1}$

In order to find $\alpha_{l+1}$, let $\alpha=\alpha_{l+1}$ and consider a scalar function of $\alpha$ :

$$
h(\alpha)=\mathcal{F}\left(\boldsymbol{x}_{l}+\alpha \boldsymbol{s}_{l+1}\right) ; \alpha \geq 0 .
$$

Since $\mathcal{F}(\boldsymbol{x}): \boldsymbol{x} \in R^{n}$ is continuously differentiable, $h(\alpha)$ is also a continuously differentiable function of $\alpha \geq 0$.

$$
\frac{d h(\alpha)}{d \alpha}=\left(\frac{\partial \mathcal{F}\left(\boldsymbol{x}_{l}+\alpha \boldsymbol{s}_{l+1}\right)}{\partial \boldsymbol{x}}\right)^{T} \boldsymbol{s}_{l+1}=\left\langle\nabla \mathcal{F}\left(\boldsymbol{x}_{l}+\alpha \boldsymbol{s}_{l+1}\right), \boldsymbol{s}_{l+1}\right\rangle
$$

thus,

$$
\left(\frac{d h(\alpha)}{d \alpha}\right)_{\alpha=0}=\left\langle\nabla \mathcal{F}\left(\boldsymbol{x}_{l}\right), \boldsymbol{s}_{l+1}\right\rangle<0
$$

Therefore, $h(\alpha)$ is monotonically decreasing function in vicinity of $\alpha \geq 0$. If $\inf \{h(\alpha): \alpha>0\}$ exists, there also exits $\alpha^{*}$ satisfying: $\alpha^{*}=\arg \min \{h(\alpha): \alpha \geq$ $0\} ; \alpha^{*}$ is called the smallest moving step.

It should be noted that if the smallest moving step $\alpha^{*}$ is chosen instead of $\alpha^{s-}$ or $\alpha^{s+}$, the searching process can be trapped at the cleft before coming the optimal point. However, choosing the moving step $\alpha^{s-}$ or $\alpha^{s+}$ ensures that the searching process runs parallel with the cleft in order to come the optimal point, as shown in Figure 2. 


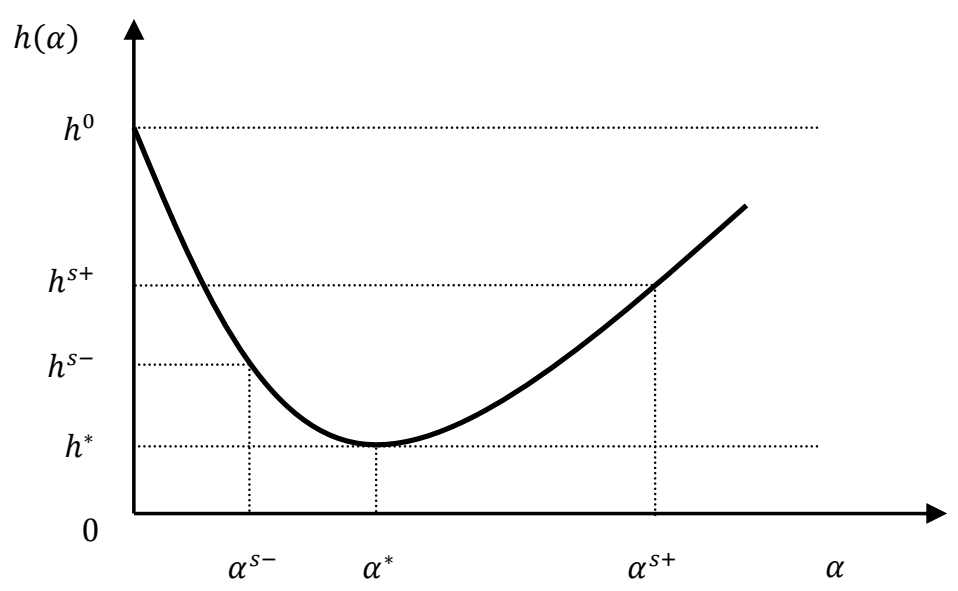

Figure 2: The Cleft-overstep principle.

The Cleft-overstep principle is to determine the moving step $\alpha^{s+} \geq \alpha^{*}$ at each iteration step and satisfy: $h^{s+}<h^{0}$, and $\frac{d h\left(\alpha^{s+}\right)}{d \alpha}>0$, as shown in Figure 2. The reason of choosing $\alpha^{s+}$ instead of $\alpha^{s-}$ is that calculation of $\alpha^{s+}$ is easier than that of $\alpha^{s-}$ by observing the change of the sign of $\frac{d h(\alpha)}{d \alpha}$ (e.g. Nguyen and Bui [8]).

\section{C) Numerical gradient calculation}

Because the function $\mathcal{F}(\boldsymbol{x})$ is treated as a black box, $\operatorname{grad}(\mathcal{F}(\boldsymbol{x}))$ only could be calculated approximately. For the approximation of $\frac{\partial \mathcal{F}(x)}{\partial x_{i}} ;(i=1,2, \ldots, n)$, let $\mathrm{y}\left(x_{i}\right)=\mathcal{F}(\boldsymbol{x})$ is a function of $x_{i}$, and the five-point method for the first derivative should be used:

$$
\frac{\partial \mathcal{F}(x)}{\partial x_{i}} \approx \frac{-\mathrm{y}\left(x_{i}+2 \Delta x_{i}\right)+8 \mathrm{y}\left(x_{i}+\Delta x_{i}\right)-8 \mathrm{y}\left(x_{i}-\Delta x_{i}\right)+\mathrm{y}\left(x_{i}-2 \Delta x_{i}\right)}{12 \Delta x_{i}}
$$

Because the parameters of running curve are in the very different ranges of value, it is crucial to choose a particular value for each of $\Delta x_{i}(i=1, \ldots, n)$. Here $\Delta x_{i}$ is chosen based a method in (e.g. Restrepo [9]).

\section{Simulation results}

The operation parameters of the train and the characteristics of the input motive force are given as Table 2 and Figure 3. 
Table 2: Operation parameters of train.

\begin{tabular}{|c|c|c|}
\hline Definition & Symbol & Value \\
\hline Total mass of train & $M[\mathrm{ton}]$ & 291.32 \\
\hline Distance & $d[\mathrm{~m}]$ & 2000 \\
\hline Inverter efficiency & $\eta$ & 0.8336 \\
\hline Gradient & $i\left[\%{ }_{00}\right]$ & - \\
\hline Running time & $T[\mathrm{~s}]$ & - \\
\hline Limit of Train Speed & $v_{\text {limit }}[\mathrm{km} / \mathrm{h}]$ & 100 \\
\hline $\begin{array}{c}\text { Limit of Train } \\
\text { Acceleration }\end{array}$ & $a_{\text {limit }}\left[\frac{\mathrm{km} / \mathrm{h}}{\mathrm{s}}\right]$ & - \\
\hline
\end{tabular}

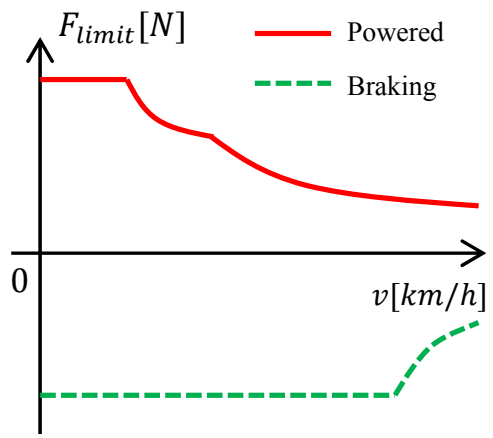

Figure 3: Characteristics of input motive force.

\subsection{Comparison of optimized running curves with different number of parameterization functions}

Simulations are carried out under the conditions: Gradient of uphill $0\left[\%{ }_{00}\right]$; the desired running time $120[s]$; no limitation of train acceleration; and no use of mechanical brakes (that means $F_{\text {mech }}(v)=0$ in all three modes of train).

With the method of choosing Parameterization functions of the running curve stated in Section 3.2, the constrained optimization problem is solved to find the parameters of these functions with 4 cases: Case 1: The number of parameters of running curves is 18; Case 2, 3, and 4: that number is respectively 24, 30, and 36 . This means that we increase the order of parameterization functions to assess the results in each case. Optimized running curves and optimized energy consumption are obtained as Figure 4(a) and Figure 4(b), respectively.

From Figure 4(a), the yellow line, black line, and green line (number of parameters of each line is 24,30 , and 36 , respectively) are very close to each other while magenta line (number of parameters is 18) is far from the other lines, which means that when the number of parameters increases, this optimized running curve gets close to a curve so-called the optimal running curve. 
From Figure 4(b), when the number of parameters increases, the optimized energy consumption decreases converges to the optimal energy consumption.

From Figure 4(a) and Figure 4(b), it is clearly seen that when the number of parameters increases (higher than 30), the optimized running curve and the optimized energy consumption do not change significantly, so the appropriate number of parameters should be 30 (Case 3). Therefore, we can obtain the parameterization functions of optimal running curve as Table 3.

Table 3: Appropriate parameterization functions of optimal running curve (Case 3: The number of parameters is 30 , and $y_{1}(x) ; y_{2}(x) ; y_{3}(x)$ are respectively parameterization functions for powered, coasting, and braking modes).

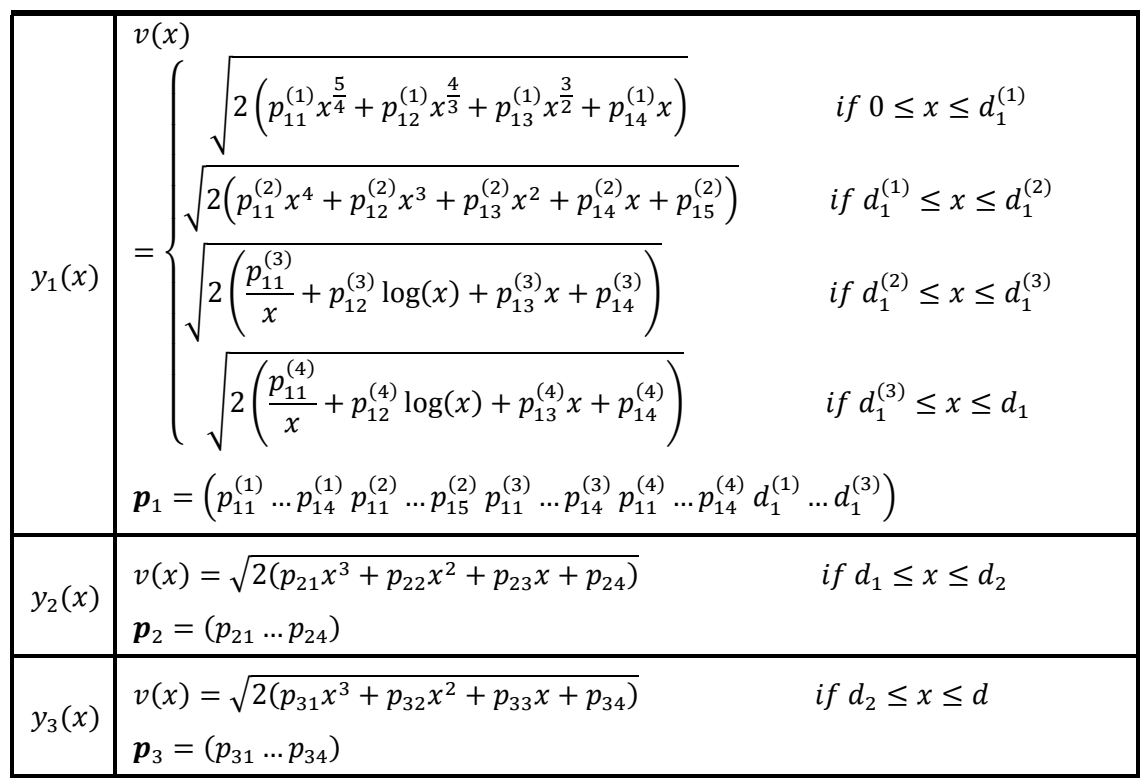

\subsection{Comparison of train operation at different running time}

In order to evaluate optimal train operation at different running time, we will find optimal running curve in each case of running time using Parameterization functions as stated in Table 3. Simulations are conducted under the same conditions as Section 6.1 except for: Gradient of uphill $2[\%]$, and the different desired running time: $116 ; 118 ; 120 ; 122 ; 124 ; 126 ; 126 ; 130 ; 135 ; 140[\mathrm{~s}]$.

Table 4 shows some simulation data including: actual running time $T_{\text {actual }}$; the notch-off speed $v_{1}$; the notch-off position $d_{1}$; the coasting-off speed $v_{2}$; the coasting-off position $d_{2}$; energy losses by resistance $E_{\text {resist }}$; and total energy consumption $E_{\text {total }}$, when the desired running times $T$ are: $116 ; 120 ; 130 ; 140[\mathrm{~s}]$. 
Table 4: Simulation data with different running time, $i=2\left[\%{ }_{00}\right]$.

\begin{tabular}{|l|l|r|r|r|r|r|}
\hline Sym. & Unit & \multicolumn{5}{|c|}{ Value } \\
\hline$T_{\text {actual }}$ & $\mathrm{s}$ & 115.9999 & 119.9999 & 126.0000 & 130.0000 & 139.9999 \\
$d_{1}$ & $\mathrm{~m}$ & 978.9083 & 708.3037 & 519.2744 & 443.3643 & 326.8848 \\
$v_{1}$ & $\mathrm{~km} / \mathrm{h}$ & 92.4539 & 85.5339 & 78.9672 & 75.6375 & 69.2231 \\
$d_{2}$ & $\mathrm{~m}$ & 1625.8000 & 1710.0000 & 1776.8000 & 1806.7000 & 1858.0000 \\
$v_{2}$ & $\mathrm{~km} / \mathrm{h}$ & 86.3708 & 75.8804 & 66.4600 & 61.7988 & 52.8890 \\
$E_{\text {resist }}$ & $\mathrm{kWh}$ & 9.2479 & 8.8805 & 8.4646 & 8.2418 & 7.8045 \\
$E_{\text {total }}$ & $\mathrm{kWh}$ & 19.1572 & 16.9222 & 15.0002 & 14.0956 & 12.4773 \\
\hline
\end{tabular}

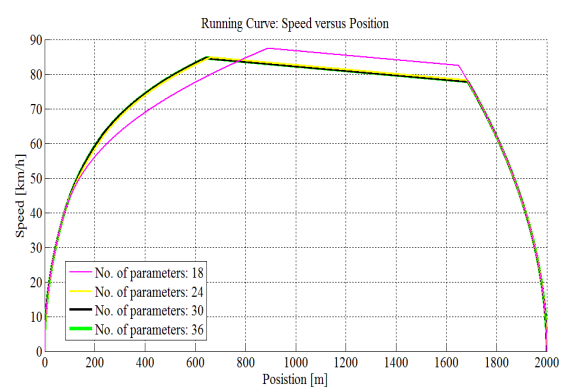

a) Optimized running curves

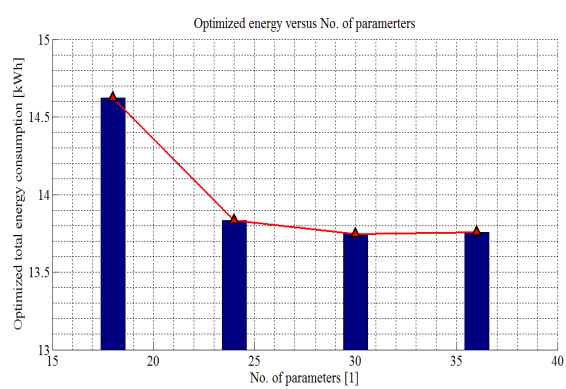

b) Optimized energy consumptions

Figure 4: Comparison of simulation results with different number of parameters. (No. of parameters of running curves in each case is respectively: $18 ; 24 ; 30 ; 36$.)

Figure 5(a) shows the optimal running curves; Figure 5(b) shows the relationship between the optimal energy consumption and the running time.

Those simulation data show that the proposed optimization method can ensure high accuracy of running time as desired. In further, we can reduce total energy consumption as well as the notch speed by increasing the running time.

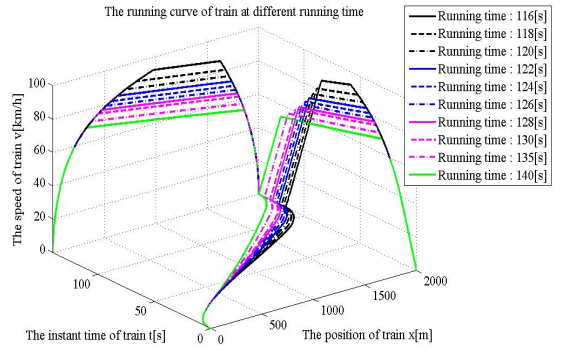

a) The optimal running curves

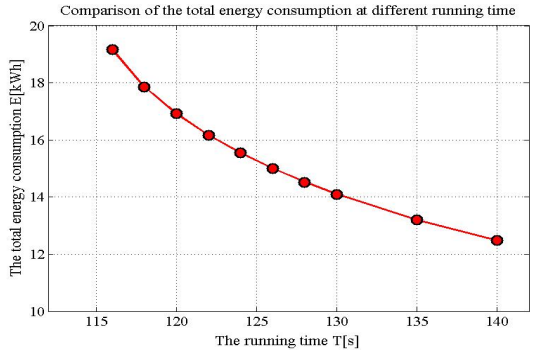

b) The optimal energy consumptions

Figure 5: $\quad$ Train operation with different running time, $i=2\left[\%{ }_{0}\right]$. 


\subsection{Comparison of train operation when train acceleration is limited for passenger's comfort}

To evaluate the effects of train acceleration limitation on the optimal train operation, simulations are carried out under the conditions: the desired running time: $122[\mathrm{~s}]$; Gradient of up-hill 2[\% $\%$ ]; no use of mechanical brakes; and three cases of train acceleration are considered: Case 1: No acceleration limitation; Case 2: $a_{\text {limit }}=2.5\left[\frac{\mathrm{km} / \mathrm{h}}{\mathrm{s}}\right]$; and Case 3: $a_{\text {limit }}=2.2\left[\frac{\mathrm{km} / \mathrm{h}}{\mathrm{s}}\right]$.

Simulation data from Table 5 and Figure 6(a) show that the train operates at higher speed when the limit of train acceleration decreases. The reason is that because the train must accelerate and decelerate more slowly, it needs running at higher speed in order to keep the running time constant.

From Figure 6(b), when the limit of train acceleration decreases, the optimal energy consumption increases, which means that if we reduce the train acceleration in Powered and Braking modes for passenger's comfort, the total energy consumption will increase. Thus, there is a trade-off between energy saving and passenger's comfort.

Table 5: Simulation data in 3 cases of train acceleration limitation. (The desired running time: $122[\mathrm{~s}]$; Gradient of uphill $i=2[\% \%]$.)

\begin{tabular}{|l|l|rrr|}
\hline \multirow{2}{*}{ Sym. } & \multirow{2}{*}{ Unit } & \multicolumn{3}{|c|}{ Value } \\
\cline { 3 - 5 } & & Case 1 & Case 2 & Case 3 \\
\hline$T_{\text {actual }}$ & $\mathrm{s}$ & 122.0000 & 121.9999 & 121.9991 \\
$d_{1}$ & $\mathrm{~m}$ & 629.9310 & 748.4832 & 1287.9000 \\
$v_{1}$ & $\mathrm{~km} / \mathrm{h}$ & 83.0486 & 86.4088 & 97.9002 \\
$d_{2}$ & $\mathrm{~m}$ & 1736.6000 & 1665.4000 & 1408.7000 \\
$v_{2}$ & $\mathrm{~km} / \mathrm{h}$ & 72.2686 & 77.6088 & 96.7767 \\
$E_{\text {resist }}$ & $\mathrm{kWh}$ & 8.7282 & 8.8891 & 9.2178 \\
$E_{\text {total }}$ & $\mathrm{kWh}$ & 16.1726 & 17.1685 & 20.9181 \\
\hline
\end{tabular}

Case 1: $a_{\text {limit }}=\infty\left[\frac{\mathrm{km} / \mathrm{h}}{\mathrm{s}}\right] ;$ Case 2: $a_{\text {limit }}=2.5\left[\frac{\mathrm{km} / \mathrm{h}}{\mathrm{s}}\right] ;$ Case $\left.3: a_{\text {limit }}=2.2\left[\frac{\mathrm{km} / \mathrm{h}}{\mathrm{s}}\right]\right)$.

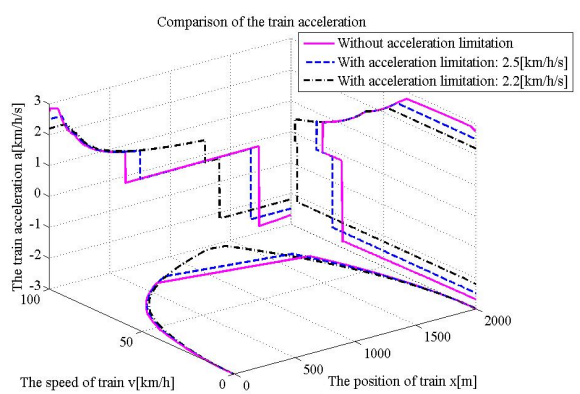

a) The optimal running curves

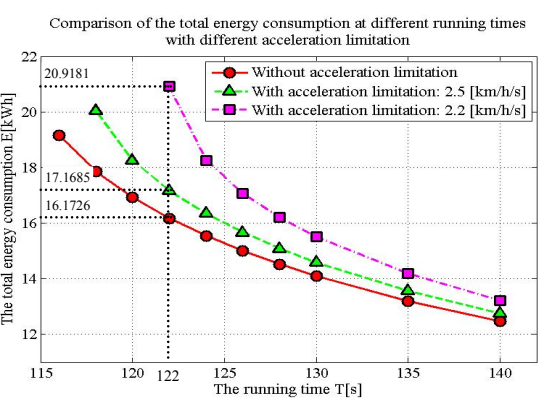

b) The optimal energy consumptions

Figure 6: Train operation in 3 cases of train acceleration limitation, $i=2[\% \%$. 


\section{Conclusions}

Due to the nonlinear model of train and the constraints of input motive force as well as that of train acceleration, etc. the speed-position relationship is complicated. Therefore, it is crucial to select parameterization functions appropriately. Section 3.2 and Section 6.1 presented a method for choosing these functions as well as the number of their parameters.

In addition, to solve the optimization problem derived in Section 4, the authors developed an effective optimization algorithm that can be applied for general optimization problems. Section 5 discussed about this algorithm.

The advantages of this research are: we can easily put additional constraints in the optimization problem: the input motive force limitation, the train acceleration limitation, the desired running time of each mode of train; no need to solve the differential equations of train at each step of searching process. Drawbacks of this research are: in case of various sectional-speed constraints, many parameterization functions should be used, which increases computation complexity; in the simulations, the authors have not taken into account the use of mechanical brakes. All these problems will be considered in future researches.

\section{References}

[1] Shaofeng Lu, Optimizing Power Management Strategies for Railway Traction Systems, Doctoral thesis, The University of Birmingham, UK, 2011.

[2] C. Chang, S. Sim, Optimizing Train Movements through Coast Control Using Genetic Algorithms, IEE Proceeding - Electric Power Applications, Vol. 144, No. 1, pp. 65-73, 1997.

[3] H.-J. Chuang, C.-S. Chen, C.-H. Lin, C.-H. Hsieh, C.-Y. Ho, Design of optimal coasting speed for saving social cost in mass rapid transit systems, Third International Conference on Electric Utility Deregulation and Restructuring and Power Technologies, pp. 2833-2839, 2008.

[4] R. Liu, L. M. Golovitcher, Energy-efficient operation of rail vehicles, Transportation Research Part A: Policy and Practice, Vol. 37, No. 10, pp. 917-932, 2003.

[5] W. Liu, Q. Li, B. Tang, Energy Saving Train Control for Urban Railway Train with Multi-Population Genetic Algorithm, International Forum on Information Technology and Applications, IEEE, IEEE Computer Society, pp. 58-63, 2009.

[6] H. Ko, T. Koseki, M. Miyatake, Application of Dynamic Programming to Optimization of Running Profile of a Train, Computer in Railway IX, Vol. 15, The Wessex Institute, pp. 103-112, 2004.

[7] Hailay Weldegiorgis Berhe, Penalty function methods using matrix laboratory (MATLAB), African Journal of Mathematics and Computer Science Research, Vol. 5(13), pp. 209-246, Nov. 2012. 
[8] V. M. Nguyen, M. T. Bui, "Method of "Cleft-Overstep" by Perpendicular Direction for Solving the Unconstrained Nonlinear Optimization Problem, Acta Mathematica Vietnamica, Vol. 15, No. 2, pp. 73-83, 1990.

[9] Ricardo Leon Restrepo, Automatic Algorithm for Accurate Numerical Gradient Calculation in General and Complex Spacecraft Trajectories, Master thesis, The University of Texas at Austin, America, pp. 31-44, Dec. 2010. 